\title{
Robustness of Supersaturated Design to Study the Causes of Medical Errors
}

\author{
Alanazi Talal Abdulrahman $\left(\mathbb{D},{ }^{1}\right.$ Abdalwahab Omar Alshammari ${ }^{(D)}{ }^{2}$ Anas Alhur $\left(\mathbb{D},{ }^{3}\right.$ \\ and Afrah Ali Alhur $\mathbb{1}^{4}$ \\ ${ }^{1}$ Department of Mathematics, University of Ha'il, Ha'il, Saudi Arabia \\ ${ }^{2}$ Department of Lab Specialist, King Khalid Hospital, Ha'il, Saudi Arabia \\ ${ }^{3}$ Department of Health Sciences, King Saud Bin Abdulaziz University, Riyadh, Saudi Arabia \\ ${ }^{4}$ Department of Clinical Nutrition, University of Ha'il, Ha'il, Saudi Arabia
}

Correspondence should be addressed to Alanazi Talal Abdulrahman; t.shyman@uoh.edu.sa

Received 16 July 2021; Accepted 27 October 2021; Published 16 November 2021

Academic Editor: Ishfaq Ahmad

Copyright (c) 2021 Alanazi Talal Abdulrahman et al. This is an open access article distributed under the Creative Commons Attribution License, which permits unrestricted use, distribution, and reproduction in any medium, provided the original work is properly cited.

\begin{abstract}
Background. In the modern contemporary, there are obvious demands for accurate interpretations of the worldwide problem, which is medication errors (MEs) due to various serious negative events that effect patient health. Most parts of the world considered health as significant issue for centuries. Recently, investigators have examined the effects of writing physician orders from the nurse's viewpoint and represent that $100 \%$ of ambiguous writing of doctor orders related directly to MEs. Objective. The aim of our work is to investigate the major causes of (MEs) in the Saudi Arabia population from multiple aspects. Methods. An online review gave quantitative information from 450 members. Respondents were heedlessly parceled into two conditions (Yes+, $\mathrm{No}-)$ and mentioned to respond to one of two plans of the explanations behind the medical errors. Fourteen determining factors in the predesign have been chosen. Entire data were collected relevant to the study purpose and the content of the questionnaire written suitably to the participants with no ambiguous terms to analyze obtained data accurately using supersaturated plans and regression methods utilizing the SPSS program to decide the real causes of the medical errors. Results. The findings indicated that often failures in the care process can be traced back to poor documentation and a lack or inadequacy of procedures; the limitations of integrated health systems between the doctor and pharmacists, human problems when standards of care, policies, processes, or procedures are not properly or effectively followed, inadequate use of technology in healthcare facilities, and unclear line of prescription from the doctor are factors that contributed to the medical errors. Conclusion. The Saudi Arabian government needs to foster a functional arrangement to examine these reasons for medical errors and make a move. Future investigations could break down the information utilizing edge plans technique.
\end{abstract}

\section{Introduction}

Most parts of the world considered health as significant issue for centuries. And nothing has been more critical than human health and a variety of decisions have been made to improve health at different rate of its effectiveness. In the modern contemporary, there are obvious demands for accurate interpretations of the worldwide problem, which is medication errors (MEs) due to various serious negative events that affect patient health. Medication error (ME) is characterized as any preventable occasion that may cause or prompt improper medicine use or patient mischief while the drug is in the control of the medical services proficient, patient, or buyer. Teoh et al. [1] reported that researchers have always seen medication errors as a universal concern in public health. According to a study performed in the UK, 5\% of the prescriptions had incorrect prescribing in terms of primary care facilities. Avery et al. [2] showed that 58\% accounted for prescription errors in Mexico, which means immediate investigation required to figure out the main 
causes and to ensure standardization for patient health. Zavaleta-Bustos et al. [3] discovered that 1,333 medication errors out of 2,380 were found demonstrating $56 \%$ of the analyzed portions and this seems to be reliable. Al-Jeraisy et al. [4] found that there are some theories on the origin of the MEs issue that have been proposed; however, there is still a need for exploring the leading causes of medication errors worldwide. Recently, investigators have examined the effects of writing physician orders from the nurse's viewpoint and represent that $100 \%$ of ambiguous writing of doctor orders are related directly to MEs. Al Khreem and Al-Khadher [5] points out that it has previously been observed in 2021 that from 504 patients analyzed 68 of their prescriptions were reported as prescription errors PEs. Recent research has assessed the formality of group pharmacists regarding biosimilar medications, which consist of 319 participants, and stated that from the total participants $39.5 \%$ illustrated a negative attitude [Anzan et al. [6]]. Alahmari et al. [7] suggested that, to minimize the number of medication errors, the health authorities in Norwegia require enhancement and wide range operating of eHealth systems to enable healthcare providers to obtain the exact and same pharmaceutical information, but for addressing the issue of medication errors there is obvious need for alteration of the workers' collaborations and working practices. Dyb and Warth [8] indicated that, in 2017, the World Health Organization (WHO) established the third Gaolable Patient Safety Challenge initiative "Medication Without Harm" to minimize preventable MEs globally by more than $50 \%$ by the coming 6 years. Srinivasamurthy et al. [9] reported that in the twenty-first century information technology plays a significant role in health field. One of the most cited studies is that of Baker [10] who sees MEs are one of the significant challenges that negatively impact patient health and patient is considered the core of all healthcare organizations. Ta'an et al. [11] indicated that MEs are profoundly common in Jordanian clinics. Over $70 \%$ of medical attendants and nursing understudies have no past preparing in forestalling and revealing MEs. The most pervasive kind of MEs was identified with changing situations for confined to bed patients followed by drug blunders, iatrogenic contaminations, and falls. Staff lack was the primary driver of MEs according to members' points of view. Bari et al. [12] reported that exhaustion because of long obligation hours, 85 (65\%), deficient experience, 66 (52\%), insufficient management, 58 (48\%) and complex case, 58 (45\%), were normal reasons for clinical blunders. Mousavi-Roknabadi et al. [13] discovered five primary components causing $\mathrm{ME}$ and underannouncing: individual elements, work environment factors, administrative elements, work culture, and blunder detailing framework.

The aim of our work is to investigate the major causes of MEs in the Saudi Arabian population from multiple aspects. An online review gave quantitative information from 450 members. Respondents were heedlessly parceled into two conditions (Yes+, No-) and mentioned to respond to one of two plans of the explanations behind the medical errors. 14 determining factors in the predesign have been chosen. Entire data was collected relevant to the study purpose and the content of the questionnaire written suitably to the participants with no ambiguous terms to analyze obtained data accurately using supersaturated plans and regression methods utilizing the SPSS program to decide the real causes of the medical errors. We begin by introducing the methodology in Section 2. In Section 3, data analysis and some examples are presented. We conclude the paper in Section 4.

\section{Method}

2.1. Robustness of Supersaturated Design. Illustrating the processes of analyzing the supersaturated designs, SSD is the purpose of this section. The direct principle impacts model is utilized in this paper:

$$
w=x \beta+\varepsilon,
$$

where $w$ is the vector of the trial's $n$ reactions, $\beta$ is the vector of unsure coefficient of $m, X$ is the $n * m$ plan framework, and it is the arbitrary mistake vector with $\left(\varepsilon_{1}, \varepsilon_{2}, \ldots\right)$ and $\varepsilon \sim N\left(0, \sigma^{2}\right)$, for $v=1,2$. Prior to introducing the models, we portray the techniques request for contrast procedure prior to introducing the models. Koukouvinos and Stylianou [14] used this method to develop a method for breaking down SSDs based on another agreement-based strategy. Suppose that, out of $(m)$ components, $p$ are dynamic. The following is the approach described by Koukouvinos and Stylianou [14]:

\section{A: figure all factor isolates through}

$$
S=X^{T} W \text {. }
$$

The reaction is $W$, and the design model lattice is $X$. Determine the pinnacle attributes and arrange factors in outright contracts at that point.

B: begin by setting $V=0$ and then using $p=1 / 2 * N$, where $N$ is the quantity of trails.

$\mathrm{C}$ : focus on the following:

$$
\mathrm{USl}_{v}=\left|s_{h}\right|-t_{h-1, \alpha / 2} \sigma_{p},
$$

and

$$
1 \mathrm{Sl}_{v}=-\left|s_{h}\right|+t_{h-1, \alpha / 2} \sigma_{p}
$$

where $t_{h-1, \alpha / 2}$ is the percent of the $t$-test.

$\mathrm{D}$ : remove the highest value $\left|s_{h-v}\right|$ and thusly set $v=v+1$.

E: using only the leftover qualities, calculate $\sigma_{p}$ for the $p$ largest supreme differences.

F: in the event that the variance in $E$ is not exactly the distinction found before $\mathrm{C}$, then, at that point conditions (3) and (4) apply. Regardless, continue to Step E and close the powerful parts from the qualifications that fall outside the crucial area.

Alanazi Talal Abdulrahman et al. [15] and Abdulrahman [16] have further details regarding this procedure. The following context is used to resolve the ethical concerns. 


\subsection{Data Collection}

2.2.1. Composed Polls and Reviews. The crucial technique is received to guarantee that included result of the investigation questions mentioned that individuals decide the reasons for drug mistakes in the Saudi Arabia populace. The outcome was broken down by utilizing the overview which is one of the notable methods for authentic information. Fourteen inquiries were spread to an assortment of members in various areas in Saudi Arabia. Four hundred and fifty people were the objective masses in the investigation.

\subsubsection{Finishing the Preliminary and Social Occasion Data.} A total of 450 individuals were divided based on gender. Eligible participates were between the ages of 15 and 80 . Table 1 clarifies the data accumulated. Information was acquired to perform evaluation and assessment of the issue of medication errors on the Saudi Arabia society.

\subsubsection{Identifying the Example and Confirming If It Suits the} Abundant Variables. Fourteen determining factors in the predesign have been chosen. Entire data were collected relevant to the study purpose and the content of the questionnaire written suitably to the participants with no ambiguous terms to analyze obtained data accurately. The design performed is analyzed implementing contrast method with both regression analysis and supersaturated designs.

\section{Data Analysis}

The ability of the contrast method to specify the active value is measured in comparison to supersaturated design methods. Abdulrahman et al. [15] and Abdulrahman [16] have further details regarding this procedure. The following context is used to resolve the ethical concerns.

Example 1. In this example, we used the $n=14$ value from Table 2 as a reference point.

The methods mentioned above of analysis have been used in sequence. Table 2 shows the results of the systems analysis for supersaturated design. All factor contrasts use (2) to calculate absolutes, which are then sorted in Table 3.

After that, starting with $V=0$, calculate the difference between the $p$ most extensive total agreements using $p=N / 2$, where $N$ denotes the number of trials. Table 4 illustrates the outcome.

We see that $\sigma_{5}^{2}$ is more than $\sigma_{4}^{2}$, and consequently we respite and find the powerful parts. The last qualities for $\mathrm{A}$ are

$$
\begin{aligned}
\mathrm{usl}_{v} & =13.52116392, \\
\operatorname{lsl}_{v} & =-13.52116392, \\
\sigma_{4}^{2} & =5.866666667
\end{aligned}
$$

Consequently, active factors $x_{9}, x_{1}, x_{11}, x_{3}, x_{5}$, and $x_{12}$ exist outside the base area. Utilizing the measurable bundle SPSS, dissect this information utilizing relapse examination (forward assessment), revealed as unique elements $x_{8}$ and $x_{11}$; a normal straight model is

$$
w=6.821-5.679 x_{8}+3.321 x_{11}+\varepsilon .
$$

The vigorous differentiation strategy brings about six dynamic variables $x_{9}, x_{1}, x_{11}, x_{3}, x_{5}$, and $x_{12}$ and the relapse examination technique brings about two dynamic components $x_{8}$ and $x_{11}$. Consequently, there is a solitary dynamic factor $x_{11}$ (often, failures in the care process can be traced back to poor documentation and a lack or inadequacy of procedures) with a straight commitment.

Example 2. In this example, similar techniques are utilized in model 1 with $n=14$; examination for contrast strategy is portrayed in Table 5 .

The arranged supreme differentiation esteems are portrayed in sort of these qualities in Table 6.

The resulting change of the $p$ greatest through and through arrangements is shown in Table 7.

The perceiving of the unique parts was ended as $\sigma_{2}^{2}$ is more than $\sigma_{1}^{2}$. The last characteristics for $\mathrm{A}$ are

$$
\begin{aligned}
\mathrm{usl}_{v} & =24.86099566 \\
\mathrm{lsl}_{v} & =-24.86099566, \\
\sigma_{1}^{2} & =61.86666667
\end{aligned}
$$

This results in seven active factors outside the essential region: $x_{13}, x_{4}, x_{6}, x_{11}, x_{12}, x_{2}$, and $x_{9}$. The unique variable ward on assessment for the present circumstance is $x_{13}$ with the going with surveyed straight model:

$$
w=9.914-6.486 x_{13}+\varepsilon .
$$

Considering the above examination, we reason that there is a working variable (the limitations of integrated health systems between the doctor and pharmacists) with a straight responsibility.

Example 3. The SSD for this model is portrayed in Table 8.

The organized all out distinction regards are shown in Table 9.

Table 10 shows what comes about on account of calculating the distinction of the $p$ greatest altogether arrangements.

What come about due to calculating the lower and uttermost cutoff focuses on essential locale are

$$
\begin{aligned}
\mathrm{usl}_{v} & =28.47273477, \\
\mathrm{lsl}_{v} & =-28.47273477, \\
\sigma_{0}^{2} & =2.666666667,
\end{aligned}
$$

which show that there are active factors outside the crucial area: $x_{3}, x_{5}, x_{8}, x_{9}$, and $x_{12}$. Assessment of this information utilizing forward determination uncovers that the force factor is $x_{8}$ and studied direct model is

$$
w=1.600+1.600 x_{8}+\varepsilon .
$$


TABle 1: Two levels in the causes of medical errors.

\begin{tabular}{|c|c|c|}
\hline Element & Sign & Explanation \\
\hline \multirow{2}{*}{ Unclear line of prescription from the doctor } & + & Yes \\
\hline & - & No \\
\hline \multirow[b]{2}{*}{ Medical abbreviations } & + & Yes \\
\hline & - & No \\
\hline \multirow{2}{*}{ Poor communication between healthcare providers } & + & Yes \\
\hline & - & No \\
\hline \multirow{2}{*}{ Poor communication between you and the doctors supervising your treatment } & + & Yes \\
\hline & - & No \\
\hline \multirow{2}{*}{ Inadequate use of technology in healthcare facilities } & + & Yes \\
\hline & - & No \\
\hline \multirow{2}{*}{ Low level of patient education to discuss and modify the pharmacist or doctor } & + & Yes \\
\hline & - & No \\
\hline \multirow{2}{*}{ Names of medicines and drugs that are similar in name or shape } & + & Yes \\
\hline & - & No \\
\hline \multirow{2}{*}{$\begin{array}{l}\text { Human problems when standards of care, policies, processes, or procedures are } \\
\text { not properly or effectively followed }\end{array}$} & + & Yes \\
\hline & - & No \\
\hline \multirow{2}{*}{ Patient issues } & + & Yes \\
\hline & - & No \\
\hline \multirow{2}{*}{ Technical malfunctions in medical devices and equipment } & + & Yes \\
\hline & - & No \\
\hline \multirow{4}{*}{$\begin{array}{l}\text { Often, failures in the care process can be traced back to poor documentation and a } \\
\text { lack or inadequacy of procedures } \\
\text { The similarity of the packaging of the preparations and the reliance on preserving } \\
\text { the external form of the drug without reading the basic information }\end{array}$} & + & Yes \\
\hline & - & No \\
\hline & + & Yes \\
\hline & - & No \\
\hline \multirow{2}{*}{ The limitations of integrated health systems between the doctor and pharmacists } & + & Yes \\
\hline & - & No \\
\hline \multirow{2}{*}{ Healthcare workers not legally aware of medication errors } & + & Yes \\
\hline & - & No \\
\hline$W$ (response) & Number & $\begin{array}{l}\text { Approximately how often do you visit a } \\
\text { healthcare facility annually? }\end{array}$ \\
\hline
\end{tabular}

TABLE 2: SSD for Example 1.

\begin{tabular}{cccccccccccccccc}
\hline$x_{1}$ & $x_{2}$ & $x_{3}$ & $x_{4}$ & $x_{5}$ & $x_{6}$ & $x_{7}$ & $x_{8}$ & $x_{9}$ & $x_{10}$ & $x_{11}$ & $x_{12}$ & $x_{13}$ & $x_{14}$ & $W$ \\
\hline+ & - & + & - & + & - & - & - & + & - & + & - & - & - & 20 \\
+ & + & + & + & + & + & + & + & + & + & + & + & + & + & 3 \\
+ & - & + & + & - & - & + & + & + & + & + & + & - & + & 5 \\
+ & + & + & + & + & + & - & + & + & + & + & + & + & + & 4 \\
+ & - & - & - & - & + & + & + & - & + & + & + & - & - & 3 \\
+ & + & + & - & - & - & + & - & + & + & - & + & + & - & 5 \\
- & + & + & + & + & + & + & + & + & - & - & + & + & + & 2 \\
+ & + & + & + & - & + & - & + & + & + & + & + & + & + & 5 \\
+ & + & - & + & + & + & + & + & + & + & + & + & + & + & 6 \\
- & - & + & + & - & - & + & + & + & + & + & - & - & - & 1 \\
- & - & - & - & - & + & - & + & + & - & + & - & - & - & 3 \\
+ & - & + & + & + & + & + & + & + & + & + & + & - & + & 6 \\
\hline
\end{tabular}

TABLE 3: Masterminded preeminent separations regards for instance 1.

\begin{tabular}{lcccccccccccccc}
\hline$j$ & 1 & 2 & 3 & 4 & 5 & 6 & 7 & 8 & 9 & 10 & 11 & 12 & 13 & 14 \\
\hline$x_{n}$ & $x_{9}$ & $x_{1}$ & $x_{11}$ & $x_{3}$ & $x_{5}$ & $x_{12}$ & $x_{2}$ & $x_{8}$ & $x_{10}$ & $x_{13}$ & $x_{4}$ & $x_{6}$ & $x_{7}$ & $x_{14}$ \\
$\left|c_{(j)}\right|$ & 57 & 51 & 49 & 39 & 19 & 15 & 13 & 13 & 13 & 13 & 1 & 1 & 1 & 1 \\
\hline
\end{tabular}

TABLE 4: Bit by bit figuring for the assessment model 1.

\begin{tabular}{ccccc}
\hline$v$ & $\sigma_{v}^{2}$ & $\mathrm{usl}_{v}$ & $\mathrm{sl}_{v}$ & $\sigma_{v}^{2}$ more than $\sigma_{v-1}^{2}$ \\
\hline 0 & 308.2666667 & 19.07574707 & -19.07574707 & \\
1 & 302.4 & 13.10794808 & -13.10794808 & No \\
\hline
\end{tabular}


TABLE 4: Continued.

\begin{tabular}{ccccc}
\hline$v$ & $\sigma_{v}^{2}$ & $\mathrm{usl}_{v}$ & $\mathrm{lsl}_{v}$ & $\sigma_{v}^{2}$ more than $\sigma_{v-1}^{2}$ \\
\hline 2 & 239.0666667 & 14.96862038 & -14.96862038 & No \\
3 & 104.6666667 & 16.20606092 & -16.20606092 & No \\
4 & 5.866666667 & 13.52116392 & -13.52116392 & No \\
5 & 26.26666667 & 3.181515653 & -3.181515653 & Yes \\
\hline
\end{tabular}

TABLe 5: SSD for Example 2.

\begin{tabular}{ccccccccccccccc}
\hline$x_{1}$ & $x_{2}$ & $x_{3}$ & $x_{4}$ & $x_{5}$ & $x_{6}$ & $x_{7}$ & $x_{8}$ & $x_{9}$ & $x_{10}$ & $x_{11}$ & $x_{12}$ & $x_{13}$ & $x_{14}$ & $W$ \\
\hline- & - & - & - & - & - & - & - & - & - & - & - & - & - & 30 \\
- & - & - & + & + & + & + & - & - & - & + & - & - & + & 8 \\
+ & + & - & + & - & - & + & - & + & + & + & + & - & + & 12 \\
- & + & + & + & + & - & + & + & + & + & + & + & + & + & 3 \\
+ & + & + & + & + & + & + & + & + & + & + & + & + & + & 0 \\
+ & + & + & + & + & + & + & + & + & + & + & + & + & + & 5 \\
+ & + & + & + & + & + & + & + & + & - & + & + & + & - & 1 \\
- & - & - & - & - & - & - & - & - & - & - & - & - & - & 2 \\
+ & + & + & + & + & + & + & + & + & + & + & + & + & + & 2 \\
+ & + & + & + & - & + & + & + & + & - & + & + & + & + & 3 \\
+ & + & + & + & + & + & + & + & + & + & + & - & - & - & 30 \\
+ & + & + & + & + & + & + & + & + & + & + & + & + & + & 10 \\
\hline
\end{tabular}

TABLE 6: Masterminded preeminent separations regards for instance 2 .

\begin{tabular}{lllllllllllllll}
\hline$j$ & 1 & 2 & 3 & 4 & 5 & 6 & 7 & 8 & 9 & 10 & 11 & 12 & 13 & 14 \\
\hline
\end{tabular}

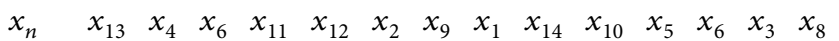
\begin{tabular}{lllllllllllllll}
$\left|c_{(j)}\right|$ & 58 & 42 & 42 & 42 & 34 & 26 & 26 & 20 & 20 & 18 & 12 & 12 & 2 & 2 \\
\hline
\end{tabular}

TABLE 7: Bit by bit figuring for the assessment model 2 .

\begin{tabular}{ccccc}
\hline$v$ & $\sigma_{v}^{2}$ & $\mathrm{usl}_{v}$ & $\mathrm{lsl}_{v}$ & $\sigma_{v}^{2}$ more than $\sigma_{v-1}^{2}$ \\
\hline 0 & 113.0666667 & 35.03211285 & -35.03211285 & \\
1 & 61.86666667 & 24.86099566 & -24.86099566 & No \\
2 & 83.86666667 & 21.84351807 & -21.84351807 & Yes \\
\hline
\end{tabular}

TABLE 8: SSD for Example 3.

\begin{tabular}{cccccccccccccccc}
\hline$x_{1}$ & $x_{2}$ & $x_{3}$ & $x_{4}$ & $x_{5}$ & $x_{6}$ & $x_{7}$ & $x_{8}$ & $x_{9}$ & $x_{10}$ & $x_{11}$ & $x_{12}$ & $x_{13}$ & $x_{14}$ & $\mathrm{~W}$ \\
\hline+ & + & + & + & + & + & + & + & + & + & + & + & - & - & 5 \\
+ & + & + & + & + & + & + & + & + & + & + & + & - & - & 5 \\
+ & + & + & + & + & - & + & + & + & - & + & + & + & + & 2 \\
+ & + & + & + & + & - & - & - & + & - & + & + & + & + & 0 \\
+ & + & + & + & + & + & + & + & + & + & + & + & - & + & 3 \\
- & - & + & + & + & + & + & - & - & + & + & - & - & - & 1 \\
+ & + & + & + & + & + & + & + & + & + & + & + & + & + & 5 \\
+ & + & + & - & + & - & + & + & + & + & + & + & + & + & 5 \\
+ & + & + & + & + & + & + & + & + & + & + & + & + & + & 3 \\
- & - & + & + & + & - & - & + & + & + & - & + & + & + & 2 \\
- & - & + & + & + & + & - & + & + & + & + & + & + & + & 1 \\
+ & + & + & + & + & + & + & + & + & - & + & + & + & + & 2 \\
\hline
\end{tabular}

The amazing contrast system achieves four powerful factors $x_{3}, x_{5}, x_{8}, x_{9}$, and $x_{12}$ while the relapse assessment procedure gives dynamic component $x_{8}$. For the present
TABLE 9: Masterminded preeminent separations regards for instance 3.

\begin{tabular}{lcccccccccccccc}
\hline$j$ & 1 & 2 & 3 & 4 & 5 & 6 & 7 & 8 & 9 & 10 & 11 & 12 & 13 & 14 \\
\hline$x_{n}$ & $x_{3}$ & $x_{5}$ & $x_{8}$ & $x_{9}$ & $x_{12}$ & $x_{1}$ & $x_{2}$ & $x_{7}$ & $x_{11}$ & $x_{10}$ & $x_{4}$ & $x_{6}$ & $x_{14}$ & $x_{13}$ \\
$\left|c_{(j)}\right|$ & 32 & 32 & 32 & 32 & 32 & 28 & 28 & 28 & 28 & 24 & 22 & 14 & 12 & 6 \\
\hline
\end{tabular}

TABLE 10: Bit by bit figuring for the assessment model 3 .

\begin{tabular}{ccccc}
\hline$v$ & $\sigma_{v}^{2}$ & $\mathrm{usl}_{v}$ & $\mathrm{lsl}_{v}$ & $\sigma_{v}^{2}$ more than $\sigma_{v-1}^{2}$ \\
\hline 0 & 2.666666667 & 28.47273477 & -28.47273477 & \\
1 & 4.266666667 & 27.49907695 & -27.49907695 & Yes \\
\hline
\end{tabular}

circumstance, there is only a solitary unique factor $x_{8}$ (human problems when standards of care, policies, processes, or procedures are not properly or effectively followed) with an immediate responsibility.

Example 4. The results from playing out a comparable framework are shown in Table 11.

By then, at that point, we discover all factor contrasts from condition 1, register the absolutes, and sort these values. The outcome is displayed in Table 12.

Start with $v=0$ and figure the distinction in the $p$ most prominent exceptional plans using $p=1 / 2 * N$, where $N$ is the quantity of fundamentals. The outcome is displayed in Table 13.

We see that $\sigma_{1}^{2}$ is more than $\sigma_{0}^{2}$, and in this way we postpone and find the incredible parts. The last attributes for A are

$$
\begin{aligned}
\mathrm{usl}_{v} & =51.18506933 \\
\mathrm{lsl}_{v} & =-51.18506933 \\
\sigma_{0}^{2} & =102
\end{aligned}
$$

Accordingly, there are active factors outside the fundamental region: $x_{1}, x_{2}, x_{3}, x_{4}, x_{5}$, and $x_{6}$. Assessment of this information dependent upon forward choice accomplishes two interesting components $x_{5}$ and $x_{14}$. The reviewed straight model:

$$
w=11.250-2.889 x_{5}-4.139 x_{14}+\varepsilon .
$$

Considering the above appraisal, we initiate that there is incredible factor (inadequate use of technology in healthcare facilities) without a prompt obligation.

Example 5. The SSD for this example is depicted in Table 14.

The organized absolute contrast regards are shown in Table 15 . 
TABLE 11: SSD for Example 4.

\begin{tabular}{ccccccccccccccc}
\hline$x_{1}$ & $x_{2}$ & $x_{3}$ & $x_{4}$ & $x_{5}$ & $x_{6}$ & $x_{7}$ & $x_{8}$ & $x_{9}$ & $x_{10}$ & $x_{11}$ & $x_{12}$ & $x_{13}$ & $x_{14}$ & $W$ \\
\hline+ & + & + & + & + & + & + & + & + & + & + & + & - & - & 10 \\
+ & + & + & + & + & + & + & + & + & - & - & + & + & + & 1 \\
+ & + & + & - & + & - & - & + & + & + & - & + & + & + & 7 \\
+ & + & + & + & + & + & - & + & + & + & + & - & + & + & 2 \\
+ & + & + & + & + & + & + & + & + & + & + & - & + & + & 5 \\
+ & + & + & + & + & - & + & - & - & - & + & + & + & + & 3 \\
+ & + & + & + & - & + & + & + & + & + & - & + & + & + & 10 \\
+ & + & + & + & + & + & + & + & - & + & + & + & + & + & 6 \\
+ & + & + & + & + & + & - & - & + & + & + & - & + & - & 15 \\
+ & + & + & + & + & + & + & + & + & + & + & + & + & + & 6 \\
+ & + & + & + & + & + & - & + & + & - & + & + & + & + & 5 \\
+ & + & + & + & + & + & + & + & - & - & - & - & - & + & 3 \\
\hline
\end{tabular}

TABLE 12: Masterminded preeminent separations regards for instance 4 .

\begin{tabular}{lcccccccccccccc}
\hline$j$ & 1 & 2 & 3 & 4 & 5 & 6 & 7 & 8 & 9 & 10 & 11 & 12 & 13 & 14 \\
\hline$x_{n}$ & $x_{1}$ & $x_{2}$ & $x_{3}$ & $x_{4}$ & $x_{5}$ & $x_{6}$ & $x_{9}$ & $x_{10}$ & $x_{13}$ & $x_{8}$ & $x_{11}$ & $x_{12}$ & $x_{14}$ & $x_{7}$ \\
$\left|c_{(j)}\right|$ & 73 & 73 & 73 & 59 & 53 & 53 & 49 & 49 & 47 & 37 & 31 & 23 & 23 & 15 \\
\hline
\end{tabular}

TABLE 13: Bit by bit figuring for the assessment model 4 .

\begin{tabular}{ccccc}
\hline$v$ & $\sigma_{v}^{2}$ & $\mathrm{usl}_{v}$ & $\mathrm{lsl}_{v}$ & $\sigma_{v}^{2}$ more than $\sigma_{v-1}^{2}$ \\
\hline 0 & 102 & 51.18506933 & -51.18506933 & \\
1 & 111.6 & 49.98084764 & -49.98084764 & Yes \\
\hline
\end{tabular}

TABLE 14: SSD for Example 5.

\begin{tabular}{ccccccccccccccc}
\hline$x_{1}$ & $x_{2}$ & $x_{3}$ & $x_{4}$ & $x_{5}$ & $x_{6}$ & $x_{7}$ & $x_{8}$ & $x_{9}$ & $x_{10}$ & $x_{11}$ & $x_{12}$ & $x_{13}$ & $x_{14}$ & $\mathrm{~W}$ \\
\hline+ & + & + & + & + & + & + & + & + & + & + & + & + & + & 6 \\
+ & + & + & + & + & + & + & + & + & + & + & + & + & + & 4 \\
+ & - & + & + & + & + & + & + & + & + & + & - & + & + & 4 \\
+ & + & + & + & + & + & + & + & + & + & + & + & + & + & 4 \\
+ & + & + & + & + & + & + & - & + & + & + & + & + & + & 10 \\
+ & + & + & + & + & + & + & + & + & + & + & + & + & + & 4 \\
+ & + & + & + & + & + & + & + & + & + & + & + & + & + & 4 \\
+ & - & - & + & + & + & - & + & + & + & + & + & + & - & 3 \\
- & + & + & - & + & + & + & + & + & + & + & + & + & - & 0 \\
+ & + & + & + & - & + & + & + & + & + & + & + & - & + & 5 \\
+ & + & + & + & + & - & + & + & + & + & - & - & - & - & 2 \\
- & + & + & + & + & + & + & + & + & + & + & + & + & + & 2 \\
\hline
\end{tabular}

Table 16 shows what comes about due to calculating the distinction of the $p$ greatest inside and out arrangements.What come about because of calculating the lower and uttermost cutoff focuses on fundamental area are

$$
\begin{array}{r}
\mathrm{usl}_{v}=43.2676771, \\
\mathrm{lsl}_{v}=-43.2676771, \\
\sigma_{0}^{2}=4.8,
\end{array}
$$

which show that there are active factors outside the major region: $x_{4}, x_{9}, x_{10}, x_{1}, x_{6}$, and $x_{11}$. Assessment of this information utilizing forward selection uncovers that the
TABLE 15: Masterminded preeminent separations regards for instance 5 .

\begin{tabular}{lcccccccccccccc}
\hline$j$ & 1 & 2 & 3 & 4 & 5 & 6 & 7 & 8 & 9 & 10 & 11 & 12 & 13 & 14 \\
\hline$x_{n}$ & $x_{4}$ & $x_{9}$ & $x_{10}$ & $x_{1}$ & $x_{6}$ & $x_{11}$ & $x_{3}$ & $x_{7}$ & $x_{5}$ & $x_{14}$ & $x_{12}$ & $x_{2}$ & $x_{13}$ & $x_{8}$ \\
$\left|c_{(j)}\right|$ & 48 & 48 & 48 & 44 & 44 & 44 & 42 & 42 & 38 & 38 & 36 & 34 & 34 & 28 \\
\hline
\end{tabular}

TABLE 16: Bit by bit figuring for the assessment model 5 .

\begin{tabular}{ccccc}
\hline$v$ & $\sigma_{v}^{2}$ & $\mathrm{usl}_{v}$ & $\mathrm{lsl}_{v}$ & $\sigma_{v}^{2}$ more than $\sigma_{v-1}^{2}$ \\
\hline 0 & 4.8 & 43.2676771 & -43.2676771 & \\
1 & 6 & 42.66256185 & -42.66256185 & Yes \\
\hline
\end{tabular}

incredible variables are $x_{1}, x_{8}$, and $x_{14}$ and studied direct model is

$$
w=5.014-1.230 x_{1}-2.84 x_{8}+973 x_{14}+\varepsilon .
$$

The amazing contrast methodology achieves four powerful factors $x_{4}, x_{9}, x_{10}, x_{1}, x_{6}$, and $x_{11}$ while the relapse assessment strategy gives dynamic components $x_{1}, x_{8}$, and $x_{14}$. For the present circumstance, there is only a solitary powerful factor $x_{1}$ (unclear line of prescription from the doctor) with an immediate responsibility.

\section{Conclusion}

Numerous actions from the outset of life had taken place to promote human health. However, medication errors create severe consequences for patient health, and they are preventable. Names of medicines and drugs are similar in name, which is one of the everlasting issues that is related with a considerable amount of the mistakes of specialists. As of late, specialists have inspected the impacts of composing doctor orders from the medical caretaker's perspective and addressed that $100 \%$ of questionable composition of specialist orders is related straightforwardly to MEs. This paper is expected to examine the significant reasons for MEs in the Saudi Arabia populace from different angles utilizing contrast technique examination and the regression investigation. An online audit gave quantitative data from 450 individuals. Respondents were carelessly divided into two conditions (Yes+, No-) and referenced to react to one of two plans of the clarifications behind the clinical mistakes. A preplan comprising of 14 elements and 12 preliminaries is chosen for execution of differentiation strategy with supersaturated plans strategies surveyed as far as their capacity to characterize the dynamic worth. The findings indicated that, often, failures in the care process can be traced back to poor documentation and a lack or inadequacy of procedures; the limitations of integrated health systems between the doctor and pharmacists, human problems when standards of care, policies, processes, or procedures are not properly or effectively followed, inadequate use of technology in healthcare facilities, and unclear writing of prescription from the doctor are factors that contributed to the medical errors. 


\section{Abbreviations}

SSD: Supersaturated designs

SPSS: Statistical Package for Social Sciences.

\section{Data Availability}

The data that support the findings of this study are available within the article and its supplementary material.

\section{Conflicts of Interest}

The authors declare that there are no conflicts of interest.

\section{Acknowledgments}

The authors are grateful to all who participated in the study. This project was funded by the Deanship of Research at the University of Ha'il, Saudi Arabia, with project no. BA-1903.

\section{Supplementary Materials}

The data regarding the details of causes of medical errors are provided. (Supplementary Materials)

\section{References}

[1] B. Teoh, A. Alrasheedy, M. Hassali, M. Tew, and M. Samsudin, "Perceptions of doctors and pharmacists towards medication error reporting and prevention in Kedah, Malaysia: a rasch model analysis," Advances in Pharmacoepidemiology \& Drug Safety, vol. 4, no. 192, pp. 2167-1052, 2015.

[2] A. A. Avery, N. Barber, M. Ghaleb et al., Investigating the Prevalence and Causes of Prescribing Errors in General Practice: The Practice Study, General Medical Council, London, UK, 2012.

[3] M. Zavaleta-Bustos, L. I. Castro-Pastrana, I. Reyes-Hernández, M. A. López-Luna, and I. B. Bermúdez-Camps, "Prescription errors in a primary care university unit: urgency of pharmaceutical care in Mexico," Revista Brasileira de Ciencias Farmaceuticas, vol. 44, no. 1, pp. 115-125, 2008.

[4] M. I. Al-Jeraisy, M. Q. Alanazi, and M. A. Abolfotouh, "Medication prescribing errors in a pediatric inpatient tertiary care setting in Saudi Arabia," BMC Research Notes, vol. 4, no. 1, pp. 1-6, 2011.

[5] S. M. Al Khreem and M. Al-Khadher, "Perceptions of nurses about medication errors: a cross-sectional study," Journal of Scientific Research in Medical and Biological Sciences, vol. 2, no. 1, pp. 30-41, 2021.

[6] M. Anzan, M. Alwhaibi, M. Almetwazi, and T. M. Alhawassi, "Prescribing errors and associated factors in discharge prescriptions in the emergency department: a prospective crosssectional study," PLoS One, vol. 16, no. 1, Article ID e0245321, 2021.

[7] A. K. Alahmari, Z. S. Almalki, M. Shahid Iqbal et al., "Knowledge and attitude of pharmacists about biosimilar medications in Saudi Arabia," International Journal of Pharmaceutical Investigation, vol. 11, no. 1, pp. 123-126, 2021.

[8] K. Dyb and L. L. Warth, "Implementing ehealth technologies: the need for changed work practices to reduce medication errors," in Proceedings of the International Conference on Informatics, Management in Technology and Healthcare (ICIMTH), pp. 83-86, Athens, Greece, 2019.
[9] S. K. Srinivasamurthy, R. Ashokkumar, S. Kodidela, S. C. Howard, C. F. Samer, and U. S. C. Rao, "Impact of computerised physician order entry (cpoe) on the incidence of chemotherapy-related medication errors: a systematic review," European Journal of Clinical Pharmacology, vol. 77, no. 8, pp. 1123-1131, 2021.

[10] A. Baker, Crossing the Quality Chasm: a New Health System for the 21st Century, British Medical Journal Publishing Group, London, UK, 2001.

[11] W. F. Ta'an, M. M. Suliman, M. M. Al-Hammouri, and A. Ta'an, "Prevalence of medical errors and barriers to report among nurses and nursing students in Jordan: a cross-sectional study," in Nursing Forum, vol. 56, pp. 284-290, Wiley Online Library, Hoboken, NJ, UK, 2021.

[12] A. Bari, R. A. Khan, and A. W. Rathore, "Medical errors; causes, consequences, emotional response and resulting behavioral change," Pakistan Journal of Medical Sciences, vol. 32, no. 3, p. 523, 2016.

[13] R. S. Mousavi-Roknabadi, M. Momennasab, M. Askarian, A. Haghshenas, and B. Marjadi, "Causes of medical errors and its under-reporting amongst pediatric nurses in Iran: a qualitative study," International Journal for Quality in Health Care, vol. 31, no. 7, pp. 541-546, 2019.

[14] C. Koukouvinos and S. Stylianou, "A method for analyzing supersaturated designs," Communications in Statistics-Simulation and Computation, vol. 34, no. 4, pp. 929-937, 2005.

[15] A. T. Abdulrahman, R. Alharbi, O. Alamri, D. Alnagar, and B. Alruwaili, "Application of supersaturated design to study the spread of electronic games," Mathematics and Statistics, vol. 9, no. 3, pp. 278-284, 2021.

[16] A. T. Abdulrahman, "Application of supersaturated design to study the actual causes of the spread of smoking," Advances and Applications in Statistics, vol. 68, no. 2, pp. 135-149, 2021. 